ARTICLE

Received 11 Mar 2013 | Accepted 21 Mar 2013 | Published 23 Apr 2013

\title{
Nanobatteries in redox-based resistive switches require extension of memristor theory
}

I. Valov ${ }^{1,2, \star}$, E. Linn ${ }^{1, \star}$, S. Tappertzhofen ${ }^{1, \star}$, S. Schmelzer ${ }^{1}$, J. van den Hurk ${ }^{1}$, F. Lentz ${ }^{2}$ \& R. Waser ${ }^{1,2}$

Redox-based nanoionic resistive memory cells are one of the most promising emerging nanodevices for future information technology with applications for memory, logic and neuromorphic computing. Recently, the serendipitous discovery of the link between redoxbased nanoionic-resistive memory cells and memristors and memristive devices has further intensified the research in this field. Here we show on both a theoretical and an experimental level that nanoionic-type memristive elements are inherently controlled by non-equilibrium states resulting in a nanobattery. As a result, the memristor theory must be extended to fit the observed non-zero-crossing $I-V$ characteristics. The initial electromotive force of the nanobattery depends on the chemistry and the transport properties of the materials system but can also be introduced during redox-based nanoionic-resistive memory cell operations. The emf has a strong impact on the dynamic behaviour of nanoscale memories, and thus, its control is one of the key factors for future device development and accurate modelling.

\footnotetext{
${ }^{1}$ Institut für Werkstoffe der Elektrotechnik II, RWTH Aachen University, 52074 Aachen, Germany. ${ }^{2}$ Peter Grünberg Institute 7, Research Centre Jülich GmbH, 52425 Jülich, Germany. ${ }^{\star}$ These authors contributed equally to this work. Correspondence and requests for materials should be addressed to I.V.

(email: i.valov@fz-juelich.de).
} 
$\mathrm{R}$ esistive switching memories are nanoionic-based electrochemical systems with a simple metal-ion conductor (insulator)-metal structure. These devices exhibit low power consumption, response times in the nanosecond range and scalability down to the atomic level ${ }^{1-3}$. They demonstrate excellent prospects for the application in modern information technology, in particular for novel logical devices ${ }^{4}$ and artificial neuromorphic systems ${ }^{5}$. The link $^{6}$ between redox-based nanoionic-resistive memories ${ }^{7}$, memristors ${ }^{8}$ and memristive devices ${ }^{9}$ has further intensified the research in this important area and inspired the introduction of new concepts ${ }^{10-13}$ and material systems ${ }^{2,3}$. Efforts are now focused on a microscopic understanding of the physicochemical processes responsible for resistive switching ${ }^{14}$ and on meeting the challenges of circuit $\operatorname{design}^{15}$.

The system properties and the material performance of redoxbased nanoionic resistive memory cell (ReRAM) devices are modulated by quantum effects, excess surface free energy of atomic clusters and nonlinear mesoscopic transport phenomena, owing to the nanodimensions of the devices in both lateral and vertical direction, that are often comparable to space charge layer lengths. The borders of well-known definitions, such as those for ion conductors and insulators, become blurred at the nanoscale, and various classes of materials starting from $\mathrm{RbAg}_{4} \mathrm{I}_{5}, \mathrm{AgI}$ (as bulk ion conductors) and continuing to $\mathrm{SiO}_{2}$ and $\mathrm{Ta}_{2} \mathrm{O}_{5}$ (as bulk insulators) are all used and termed ionic or mixed ionic-electronic electrolytes at room temperature ${ }^{16-20}$.

The cation-migration-based electrochemical metallization memory (ECM) cells are a class of ReRAMs that use $\mathrm{Ag}$ or $\mathrm{Cu}$ as an active electrode and, for example, $\mathrm{Pt}$, Ir or $\mathrm{W}$ as an inert counter electrode. A variety of oxide, chalcogenide and halide thin films have been suggested for the solid electrolyte ${ }^{2}$. Applying a positive voltage between the active and the counter electrode leads to an oxidation (dissolution) of the active electrode's material and to a deposition of metal $(\mathrm{Ag}$ or $\mathrm{Cu})$ at the counter electrode. Owing to the high electric field in the order of $10^{8} \mathrm{~V} \mathrm{~m}^{-1}$, the metallic deposit propagates in a filamentary form and short circuits the cell, thus defining a low-resistive ON state. The filament can be dissolved by applying a voltage of opposite polarity to return the cell to a high-resistive OFF state. The anion-migration-based valence change cells (VCM) typically use a high work function electrode (for example, Pt and TiN), an oxygen-affine, lower work function electrode and a metal oxide as the electrolyte. These cells rely on the formation of oxygen-deficient, mixed ionic-electronic conducting filaments and the nanoionic modification of the potential barrier between the tip of the filament and the electrode to define the $\mathrm{ON}$ and OFF states. The ON and OFF states are then used to read the Boolean 1 and 0 , respectively.

The functionality of ReRAM cells and the kinetics of the filament formation/dissolution are the subject of intensive studies from both, academia and industry, leading to a development of empirical or semi-empirical models for the operating principles ${ }^{21}$, expanding to include concepts of multibit memories ${ }^{22}$ and memristive systems $6,10,15$.

From the circuit theory's point of view, ReRAM cells are regarded as memristive elements or real memristors ${ }^{23}$. They are defined by two simple equations, the state-dependent Ohm's law,

$$
I=G(\mathbf{x}, V) \cdot V,
$$

and the state equation

$$
\frac{\mathrm{d} \mathbf{x}}{\mathrm{d} t}=\mathrm{f}(\mathbf{x}, V)
$$

The distinctive feature of memristive elements is a pinched characteristics at the origin of the $I-V$ plane (that is, the $I-V$ zerocrossing property $)^{8,9}$, which is a direct result of these equations, and therefore represents the essential fingerprint ${ }^{23}$. A short excursus on the use of the term 'memristor' is given in Supplementary Note 1 .

Here, we report on non-equilibrium $\mathrm{ON}$ and OFF states in ReRAM cells determined by chemical potential gradients generating an electromotive force of up to a few hundred millivolts, violating the zero-crossing property. The emf may affect the retention time, and both influences and is influenced by the processes during formation and rupture of the metallic filament. We introduce additional equations to account for the emf, that is, for the non-zero-crossing hysteresis loop, thus qualitatively and quantitatively extending the memristor theory. The conclusions we draw with respect to a series of ECM systems apply to VCM-type ReRAM cells as well as other electrochemical and (neuro-)biological systems.

\section{Results}

Origins of electromotive force in ReRAM cells. To clearly identify the individual influences of different chemical potential gradients, we studied ECM cells build from a series of materials, selected so as to ensure a transition of particular chemical and transport properties, that is, $\mathrm{SiO}_{2}-\mathrm{GeS}_{x}, \mathrm{GeSe}_{x}-\mathrm{AgI}$ (with $x=2.2$ and 2.3 , respectively). In the as-deposited state, all materials are electronic insulators where the first and the last compounds of this series represent the two extremes. That is to say, $\mathrm{SiO}_{2}$ has a very small (but at the nanoscale not completely negligible) electronic conductivity and AgI is a stoichiometric ionic compound with considerable $\mathrm{Ag}^{+}$ion conductivity. Neither is able to dissolve Ag chemically. In contrast, $\mathrm{GeS}_{x}$ and $\mathrm{GeSe}_{x}$ are able to dissolve $\mathrm{Ag}$ ( or $\mathrm{Cu}$ ) to different extents moving from insulators to mixed ionic-electronic electrolytes, thus displaying a transport property transition between $\mathrm{SiO}_{2}$ and AgI.

We found three factors that contribute to the formation of the cell voltage $V_{\text {emf }}$, as illustrated in Fig. 1: (1) the classical Nernst potential $V_{\mathrm{N}}$ (Fig. 1a), (2) the diffusion potential $V_{\mathrm{d}}$ (Fig. 1b) and (3) the Gibbs-Thomson potential due to the different surface free energies of macro- and nanoparticles $V_{\mathrm{GT}}$ (Fig. 1c), for the case that a metallic nanofilament is formed without shortcircuiting the electrodes. Note that in the case that a metallic nanofilament is formed, the measureable emf is zero owing to the short circuit (Fig. 1d).

The Nernst voltage $V_{\mathrm{N}}$ is given by the difference between the potential-determining half-cell reactions at each electrode/electrolyte interface:

$$
V_{\mathrm{N}}=V_{\mathrm{s}^{\prime}}-V_{\mathrm{s}^{\prime \prime}}=V^{0}+\frac{k T}{z e} \ln \frac{\left(a_{\left.\mathrm{Me}^{z}+\right)_{\mathrm{s}^{\prime}}} \cdot\left(a_{\mathrm{Red}}\right)_{\mathrm{s}^{\prime \prime}}\right.}{\left(a_{\mathrm{Me}}\right)_{\mathrm{s}^{\prime}} \cdot\left(a_{\mathrm{Ox}}\right)_{\mathrm{s}^{\prime \prime}}}
$$

with $V_{\mathrm{s}^{\prime}}$ and $V_{\mathrm{s}^{\prime \prime}}$ being the half-cell potentials at the active electrode/electrolyte $\left(s^{\prime}\right)$ and inert electrode/electrolyte $\left(s^{\prime \prime}\right)$ interfaces, $z$ the number of exchanged electrons and $V^{0}$ the difference in the standard potentials of these reactions. At the $s^{\prime}$ interface, the potential-determining reaction is the same for all ECM cells:

$$
\mathrm{Me}^{z+}+z e^{-} \rightleftharpoons \mathrm{Me}
$$

At the $s^{\prime \prime}$ interface, Red and Ox are general expressions in equation (3) for a species undergoing a redox process. The nature of this species is determined by the specific cell, as will be shown below.

The non-equilibrium diffusion potential $V_{\mathrm{d}}$ in ECM cells arises owing to excess concentrations of charged species, that is, $\mathrm{Ag}^{+}$ ions, electrons and/or $\mathrm{OH}^{-}$ions within the electrolyte film. These ions are introduced into the solid films either electrochemically during forming or SET/RESET cycles or chemically owing to a chemical dissolution of Ag. In both cases, the ratio of 
concentration changes across the electrolyte layer, with particularly pronounced changes in the vicinity of the electrodes. The electromotive force generated by this inhomogeneous charge distribution and mobilities is given by ${ }^{24}$ :

$$
V_{\mathrm{d}}=-\frac{k T}{e} \sum_{i} \int_{\mathrm{s}^{\prime \prime}}^{\mathrm{s}^{\prime}} \frac{t_{i}}{z_{i}} d \ln a_{i}=-\frac{k T}{e}\left(\bar{t}_{\mathrm{Me}^{+}} \ln \frac{\left(a_{\mathrm{Me}^{+}}\right)_{\mathrm{s}^{\prime}}}{\left(a_{\left.\mathrm{Me}^{+}\right)_{\mathrm{s}^{\prime \prime}}}\right.}-\bar{t}^{-} \ln \frac{\left(a^{-}\right)_{\mathrm{s}^{\prime}}}{\left(a^{-}\right)_{\mathrm{s}^{\prime \prime}}}\right)
$$

where $k, T$ and $e$ are the Boltzmann constant, the temperature and the elementary charge, respectively. $\overline{\mathrm{Me}}_{\mathrm{Me}^{+}}$and $a_{\mathrm{Me}^{+}}$denote the averaged transference number and the activity of the metal cations, respectively. The averaged transference number and the activity of negatively charged species (anions and electrons) are expressed by $\bar{t}^{-}$and $a^{-} \cdot z_{i}$ is the charge number of each species. The interfaces denoted by $s^{\prime}$ and $s^{\prime \prime}$ correspond to the active electrode/electrolyte and the inert electrode/electrolyte interfaces, respectively.

The potential difference generated in the neuron cells to transport electric signals has the same nature and originates in the diffusion (Donnan) potential ${ }^{24}$.

The contribution of the third component $V_{\mathrm{GT}}$ to the total cell voltage $V_{\text {emf }}$ is expected in the case of a non-contacting filament

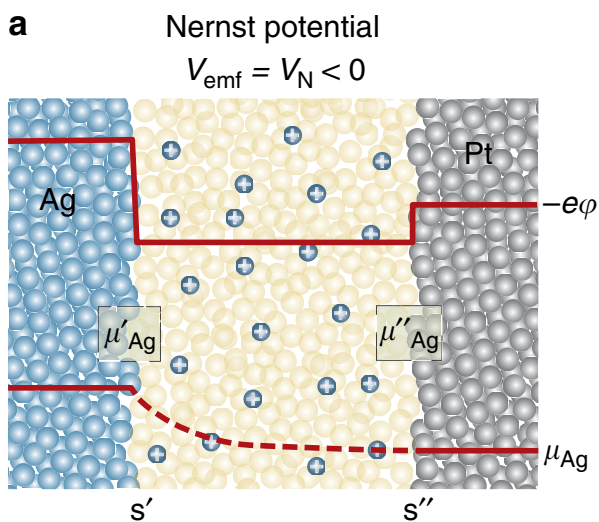

c Gibbs-Thomson effect

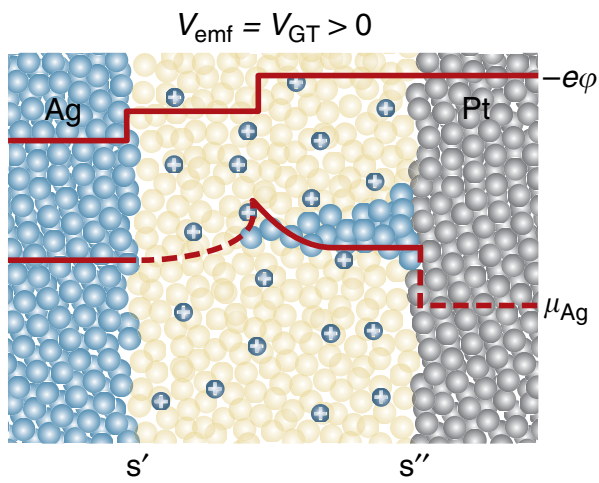

owing to the different surface free energies of the macrocrystalline active electrode and the nanosize filament in accordance with the Gibbs-Thomson equation:

$$
V_{\mathrm{GT}}=-\frac{\mu_{\mathrm{Ag}}^{\mathrm{macro}}-\mu_{\mathrm{Ag}}^{\mathrm{nano}}}{z e}=-\frac{2 \gamma}{z e r} V_{\mathrm{m}},
$$

where $\gamma$ is the surface free energy, $r$ is the radius of the particle, $V_{\mathrm{m}}$ is the molar volume and $\mu_{\mathrm{Ag}}$ is the chemical potential of $\mathrm{Ag}$. $V_{\mathrm{GT}}$ can be observed, for instance, for high-resistive $(R>12.9 \mathrm{k} \Omega)$ ON states (no metallic short circuit) typically caused by filaments in ECM cells without galvanic contact to the active electrode (see Supplementary Fig. S1). It should be noted that the contribution of $V_{\mathrm{GT}}$ could not be clearly distinguished from the contribution of $V_{\mathrm{d}}$ in the particular systems we have studied.

ReRAM cells based on initially insulating thin films. In Fig. 2, we show the time evolution of the open cell voltage $V_{\text {cell }}$ and of the short circuit current $I$ for $\mathrm{Ag} / \mathrm{SiO}_{2} / \mathrm{Pt}$ cells. Figure 2a displays the simplified equivalent circuit of the nanobattery where the cell voltage is

$$
V_{\text {cell }}=t_{\text {ion }} V_{\text {emf }}=\frac{R_{\mathrm{i}}^{-1}}{R_{\mathrm{i}}^{-1}+R_{\mathrm{e}}^{-1}} V_{\mathrm{emf}}
$$
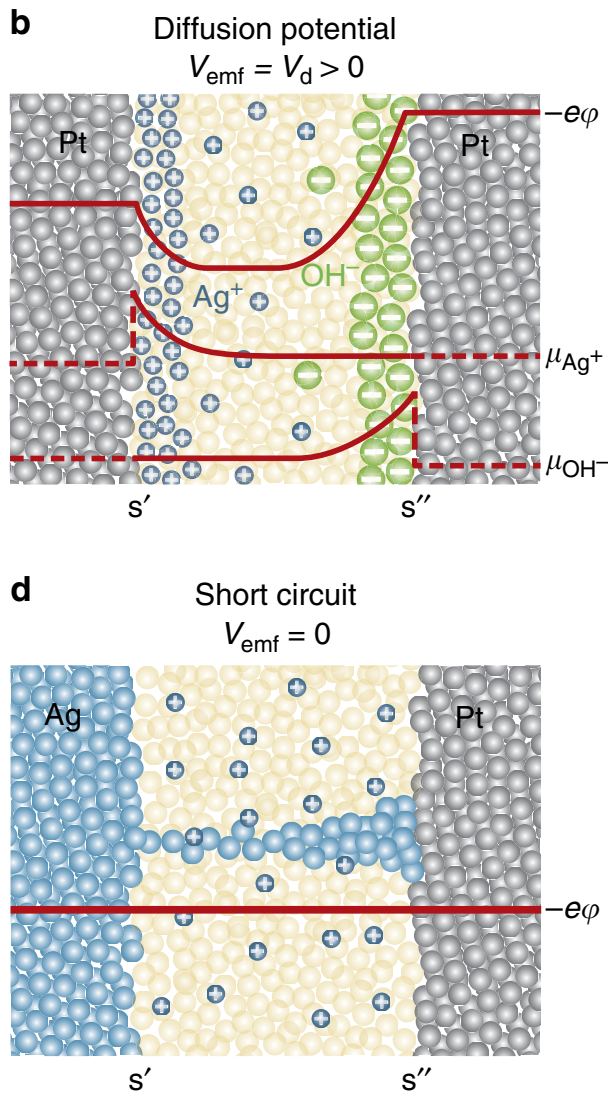

Figure 1 | Origins of emf in nanoscale cells. These sketches show situations for $\mathrm{SiO}_{2}$-based cells in which one of the three basis origins of emf dominates. (a) A Nernst potential $V_{\mathrm{N}}$ of a $\mathrm{Ag} / \mathrm{SiO}_{2} / \mathrm{Pt}$ cell arising from the difference of the chemical potential of $\mathrm{Ag}$ metal at the interfaces $\mathrm{Ag} / \mathrm{electrolyte}$ and $\mathrm{Pt} /$ electrolyte $\Delta \mu_{\mathrm{Ag}}=\mu_{\mathrm{Ag}}^{\prime}-\mu^{\prime \prime}$ Ag. The Nernst potential $V_{N}$ according to equation (11) has a negative value and it is given by the difference of the electrical potentials at both electrodes $\Delta \varphi=V_{\mathrm{emf}}=-\Delta \mu_{\mathrm{Ag}}$ /ze generated to keep the condition $\sum_{\mathrm{i}} \tilde{\mu}_{\mathrm{i}}=0\left(\tilde{\mu}_{\mathrm{i}}\right.$ is the electrochemical potential given by $\left.\tilde{\mu}_{\mathrm{i}}=\mu_{\mathrm{i}}+z e \varphi\right)$. Depending on the specific chemical redox system and the chemical potential gradients, the sign of the $V_{\text {emf }}$ can also be positive. (b) A diffusion potential $V_{\mathrm{d}}$ is generated in a $\mathrm{Pt} / \mathrm{SiO}_{2} / \mathrm{Pt}$ cell by gradients of the chemical potentials of the $\mathrm{Ag}{ }^{+}$and $\mathrm{OH}^{-}$ions, that is, $\Delta \mu_{\mathrm{Ag}^{+}}=\mu^{\prime} \mathrm{Ag}^{+}-\mu^{\prime \prime} \mathrm{Ag}^{+}$and $\Delta \mu_{\mathrm{OH}^{-}}=\mu_{\mathrm{OH}^{-}}^{\prime}-\mu_{\mathrm{OH}}^{\prime \prime}$ inhomogeneously distributed in the thin film as given by equation (5). (c) In the case of a nanosize filament, the chemical potential of $\mathrm{Ag}$ contains an additional surface energy term generating a chemical potential gradient $\Delta \mu_{\mathrm{Ag}}=\mu_{\mathrm{Ag} \text {-micro }}-\mu_{\mathrm{Ag} \text {-nano }}$ in accordance with equation (6) (Gibbs-Thomson Potential $V_{\mathrm{GT}}$ ). (d) In the case of a fully metallic contact or a highly conducting tunnel junction, the emf is $V_{\text {cell }}=0$. The potential of the right electrode is used as a reference throughout the text. Please note that profiles of the electrostatic potential $\varphi$ is sketched without zooming into the space charge layers. 
a

Equivalent circuit model
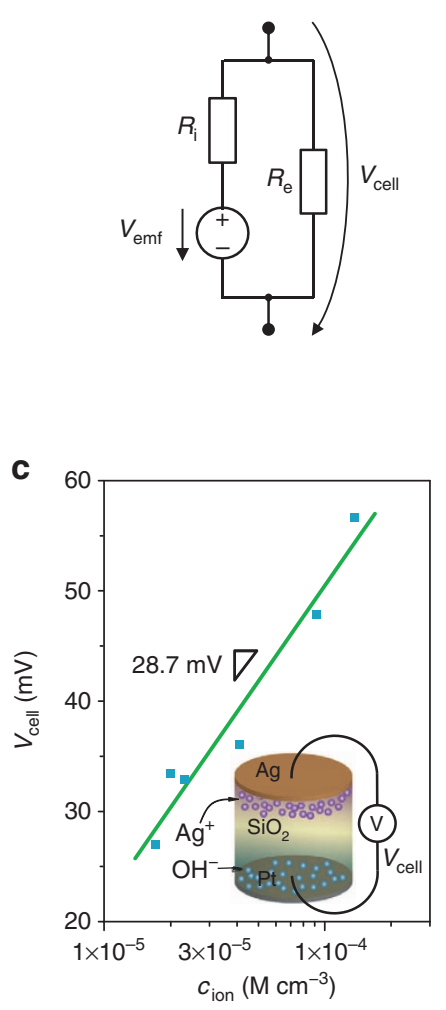

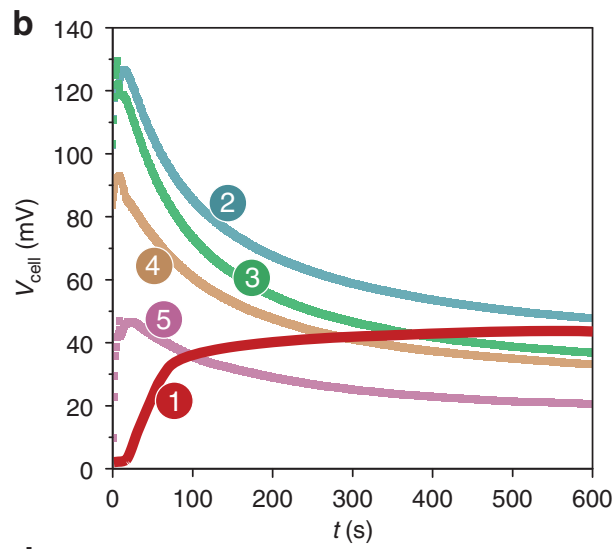

d

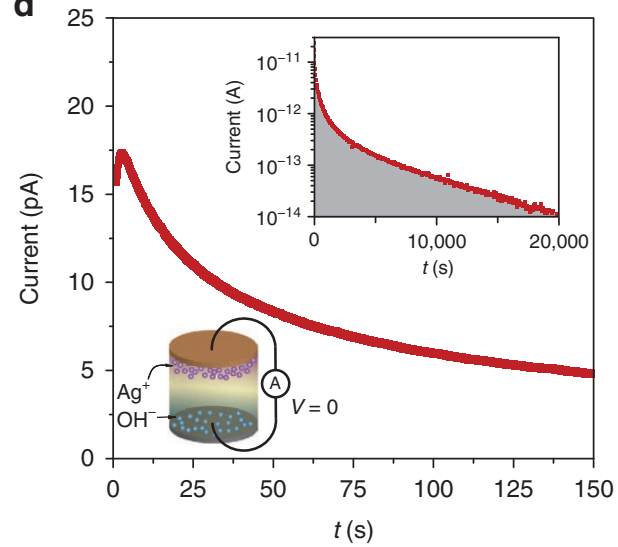

Figure 2 | Steady-state emf measurements for $\mathbf{A g} / \mathbf{S i O}_{2} / \mathbf{P t ~ E C M}$ cells. (a) Simplified equivalent circuit model of a $\mathrm{ReRAM}$ device. (b) $V_{\text {cell }}$ for a $\mathrm{Ag} / \mathrm{SiO} / 2$ Pt cell measured under open circuit conditions. The red line curve (1) depicts $V_{\text {cell }}$ in the OFF state after a SET/RESET cycle. For the other curves, the ion concentration $c_{\text {ion }}$ (that is, the sum of the $\mathrm{Ag}^{+}$and $\mathrm{OH}^{-}$ion concentrations, averaged over the thickness) was controlled and preset using different sweep rates ${ }^{25}$. Curve (2) corresponds to $c_{\text {ion }}=1.4 \times 10^{-4} \mathrm{M} \mathrm{cm}^{-3}$, (3) to $c_{\text {ion }}=9.2 \times 10^{-5} \mathrm{M} \mathrm{cm}^{-3}$, (4) to $c_{\text {ion }}=2 \times 10^{-5} \mathrm{M} \mathrm{cm}^{-3}$ and curve (5) to $c_{\text {ion }}=1.7 \times 10^{-5} \mathrm{M} \mathrm{cm}^{-3}$, respectively. Details of the measurements are depicted in Supplementary Fig. S2. Further voltage sweeps in the negative voltage regime result in further decrease of the ion concentration (Supplementary Fig. S3). (c) The slope of the line provides the pre-exponential term and we were thus able to determine the ionic transference number $\bar{t}_{\text {ion }}$ by using equations (10) and $z=+1$ (for $\mathrm{Ag}^{+}$). (d) The time evolution of the discharge current (for $V=0 \mathrm{~V}$ ) of the cell (diameter $d=100 \mu \mathrm{m}$ ) after SET and subsequent RESET operation is shown. Inset: the same plot for an extended time and a log current scale. The integration reveals the charge $(5 \mathrm{nC})$ of the nanobattery.

Here, $t_{\text {ion }}$ represents the total ionic transfer number, $R_{\mathrm{i}}$ is the total resistance of the ionic current path and $R_{\mathrm{e}}$ the total electronic resistance of the cell.

Figure $2 \mathrm{~b}$ shows $V_{\text {cell }}$ after establishing a defined concentration $c_{\text {ion }}$ of $\mathrm{Ag}^{+}$and $\mathrm{OH}^{-}$ions for unformed cells. We utilized the fact that the amount of ions generated at the $s^{\prime}$ and $s^{\prime \prime}$ interfaces during cell operation is adjusted by the pulse length and height or the sweep rate ${ }^{25}$. The decrease of $V_{\text {cell }}$ over a few hundred seconds is caused by the equilibration of the $\mathrm{Ag}^{+}$and $\mathrm{OH}^{-}$concentration gradient, which has an initial maximum immediately at the metal/ electrolyte interface. Furthermore, Fig. 2b (red line) shows $V_{\text {cell }}$ immediately after a RESET operation and switching to the open cell measuring conditions. In this case, the $\mathrm{Ag}^{+}$concentration in the immediate vicinity to the Ag electrode is depleted (in contrast to unformed cells) on the one hand side owing to the reduction process (that is, the RESET process) and on the other hand side owing to slow supply/diffusion of ions from layers apart from the interface (diffusion limited). Thus, in this situation $V_{\text {cell }}$ increases with time but relaxes to the same value as the unformed cells after equilibration.

The potential-determining reaction at the inert electrode, for example, $\mathrm{Pt}$, depends on the material properties of the solid electrolyte and we distinguish two cases. The system $\mathrm{Ag} / \mathrm{SiO}_{2} / \mathrm{Pt}$ represents the first case for which the solid $\mathrm{SiO}_{2}$ film contains no initial $\mathrm{Ag}^{+}$and, hence, reaction (4) cannot be potentialdetermining. Instead, as shown in Tsuruoka et al. ${ }^{26}$, moisture is typically incorporated into this electrolyte during fabrication and hence protons provide the required counter reaction at the inert electrode, for example,

$$
\begin{aligned}
& \frac{1}{2} \mathrm{O}_{2}+\mathrm{H}_{2} \mathrm{O}+2 e^{-} \rightleftharpoons 2 \mathrm{OH}^{-} \\
& \quad\left(\text { or alternatively } 2 \mathrm{H}_{2} \mathrm{O}+2 e^{-} \rightleftharpoons 2 \mathrm{OH}^{-}+\mathrm{H}_{2}\right)
\end{aligned}
$$

and the Nernst voltage takes the form:

$$
V_{\mathrm{N}}=V^{0}+\frac{k T}{2 e} \ln \frac{\left(a_{\mathrm{Me}^{z+}}^{2}\right)_{\mathrm{s}^{\prime}} \cdot\left(a_{\mathrm{OH}^{-}}^{2}\right)_{\mathrm{s}^{\prime \prime}}}{\left(a_{\mathrm{Me}}^{2}\right)_{\mathrm{s}^{\prime}} \cdot\left(a_{\mathrm{O}_{2}}^{\frac{1}{2}}\right)_{\mathrm{s}^{\prime \prime}} \cdot\left(a_{\mathrm{H}_{2} \mathrm{O}}\right)_{\mathrm{s}^{\prime \prime}}}
$$

The total emf of the ECM cell is a combination of equations (5) and (9) and is expressed by:

$$
\begin{aligned}
V_{\mathrm{emf}}= & V_{\mathrm{N}}+V_{\mathrm{d}}=V_{0}+\bar{t}_{\mathrm{OH}^{-}} \frac{k T}{e} \ln \left(a_{\mathrm{Me}^{+}}\right)_{\mathrm{s}^{\prime}} \\
& +\bar{t}_{\mathrm{Me}^{+}} \frac{k T}{e} \ln \left(a_{\mathrm{OH}^{-}}\right)_{\mathrm{s}^{\prime \prime}} \sim V_{0}+\bar{t}_{\mathrm{ion}} \frac{k T}{2 e} \ln \left(a_{\mathrm{ion}}\right)
\end{aligned}
$$

with $V_{0}=V^{0}+$ const. (see Supplementary Note 2). 
In Fig. $2 c$ we evaluate $V_{\text {cell }}$ after initial relaxation. The slope of the line provides the pre-exponential term and we were thus able to determine the ionic transference number $\bar{t}_{\text {ion }}=\bar{t}_{\mathrm{Ag}^{+}}+\bar{t}_{\mathrm{OH}^{-}}=0.4$ by using equation (10). The $x$-axes intercept corresponds to the condition $\ln \left(a_{\text {ion }}\right)=0$ and provides the value of $V_{0}=0.17 \mathrm{~V}$. Both values are used as parameters in the device modelling below.

Figure 2d illustrates the short-circuit currents of a Ag/SiO $/ 2$ Pt cell after RESET operation. In the inset, an extended discharge time of $20,000 \mathrm{~s}(\sim 5.5 \mathrm{~h})$ is shown. The measurements were carried out until they approached the resolution limit of our measuring system ( $\sim 10 \mathrm{fA}$ ). The current is proportional to the electrode area (see Supplementary Fig. S4). An integration of current over time reveals a total charge of $\sim 5 \mathrm{nC}$ released during the discharge of the battery, which corresponds to a conversion of $\sim 2$ monolayers Ag. In comparison, the dielectric discharge of the cell capacitor is $\sim 0.4 \mathrm{pC}$, clearly demonstrating that the discharge phenomenon is of an electrochemical nature.

Please note that retention time of the cell (here: in the OFF state) is not immediately related to the relaxation time of the emf voltage. While the first has to be $>10$ years for universal non-volatile memories, the latter may be in the order of minutes to days as shown. In other words, the discharge of the ReRAM nanobattery does not lead to a loss of the OFF state (in a similar manner as the discharge of any other rechargeable battery does to end up in a filamentary short between the electrodes). Instead, typically only a shift of the particular $R_{\mathrm{OFF}}$ value is observed upon the relaxation of the emf. Such a shift has been reported, for example, by Miao et al. ${ }^{27}$ and Choi et al. ${ }^{28}$ for both ECM and VCM systems, respectively. More detailed discussion is provided in Supplementary Note 3.

ReRAM cells based on mixed conducting thin films. In the second case condition for establishing a Nernst voltage according to equation (3), the electrolyte contains mobile $\mathrm{Me}^{z+}$ ions dissolved by electrochemical and/or chemical processes with almost homogeneous distribution, that is, $\left(a_{\mathrm{Me}^{z+}}\right)_{\mathrm{s}^{\prime}} \sim\left(a_{\mathrm{Me}^{z+}}\right)_{\mathrm{s}^{\prime \prime}}$. Then the potential-determining half-cell reaction at the $\mathrm{s}^{\prime \prime}$ interface will be the same as at $s^{\prime}$, that is, reaction (4), and, because no chemical potential gradient of the charged species is assumed, equation (3) can be simplified to:

$$
V_{\mathrm{emf}}=\bar{t}_{\text {ion }} \frac{k T}{e} \ln \frac{\left(a_{\mathrm{Me}}\right)_{\mathrm{s}^{\prime \prime}}}{\left(a_{\mathrm{Me}}\right)_{\mathrm{s}^{\prime}}}
$$

where $\left(a_{\mathrm{Me}}\right)_{\mathrm{s}^{\prime}}$ denotes the activity of the active metal Me at interface $s^{\prime}$, that is, $\left(a_{\mathrm{Me}}\right)_{\mathrm{s}^{\prime}}=1$, if a pure metal is used, and $\left(a_{\mathrm{Me}}\right)_{\mathrm{s}^{\prime \prime}}$ is the activity of Me at the inert counter electrode $s^{\prime \prime}$, that is, $\left(a_{\mathrm{Me}}\right)_{s^{\prime \prime}}$ is typically very low. $\bar{t}_{\text {ion }}$ is the ion transference number averaged throughout the electrolyte thickness. As $\left(a_{\mathrm{Me}}\right)_{\mathrm{s}^{\prime}}$ is fixed, the Nernst voltage is a function of $\left(a_{\mathrm{Me}}\right)_{\mathrm{s}^{\prime \prime}}$ alone. We succeeded in demonstrating a Nernst emf in accordance with equation (11) (Fig. 1a) for the systems $\mathrm{Ag} / \mathrm{Ag}-\mathrm{GeS}_{2.2} / \mathrm{Pt}$ and $\mathrm{Ag} / \mathrm{Ag}-\mathrm{GeSe}_{2.3} / \mathrm{Pt}$, which contain a significant amount of chemically dissolved $\mathrm{Ag}^{+}$ ions (a solubility of up to 35 at $\% \mathrm{Ag}$ has been reported ${ }^{29}$ ). We applied a positive external voltage of $1 \mathrm{~V}$ to the inert electrode, thus removing the residual $\mathrm{Ag}$ atoms from the $\mathrm{s}^{\prime \prime}$ interface and lowering their activity. In the coupled cathodic reaction at the Ag interface s', Ag dendrites are deposited as shown in the optical image in Fig. 3. Thus, apart from the different concentration of $\mathrm{Ag}^{+}$ions at both interfaces, we generated a difference in the activities of Ag. The emf of the system was then monitored over time and correlated to the evolution of the dendrite morphology further recorded by optical images. The time evolution shown in Fig. 3 arises from the interplay of a Nernst potential and a diffusion potential. In the voltage-modified state $\left(t_{1}\right.$ in Fig. 3$)$, the contribution of the (positive) diffusion potential fades, leading to a dominating (negative) Nernst potential, which results to an emf
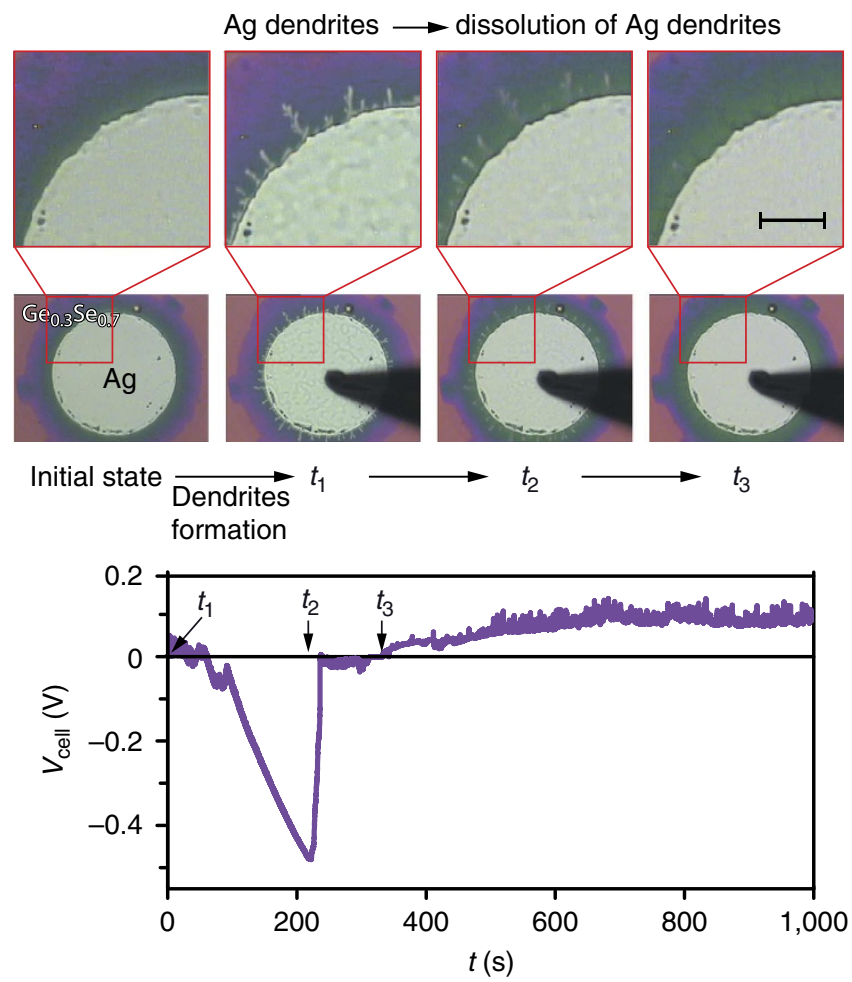

Figure 3 | Time-dependent emf measurements for $\mathrm{Ag} / \mathrm{Ge}_{0.3} \mathrm{Se}_{0.7} / \mathrm{Pt}$ ECM cells. After a SET/RESET cycle (initial state), a negative voltage is applied to the $\mathrm{Ag}$ electrode resulting in a dendrite formation. At $t_{1}=0 \mathrm{~s}$ the voltage measurement is started. Fading of the diffusion potential contribution from $t_{1}$ to $t_{2}$ leads to a highly negative $V_{\text {cell }}$ owing to a significant Nernst potential between the electrodes. In parallel to the dissolution of the dendrites $\left(t_{2}\right)$, the emf increases to positive values again with a diffusion potential as the remaining component of the emf owing to the different activity of $\mathrm{Ag}^{+}$ions at the both $\mathrm{s}^{\prime}, \mathrm{s}^{\prime \prime}$ surfaces $\left(t>t_{3}\right)$. The scale bar in the inset corresponds to $20 \mu \mathrm{m}$.

value of $\sim-450 \mathrm{mV}$ owing to the contribution of equation (11). The emf value corresponds to a difference in the Ag activities at both electrodes, $\left(a_{\mathrm{Ag}}\right)_{\mathrm{s}^{\prime \prime}} \approx 2.5 \times 10^{-8}\left(a_{\mathrm{Ag}}\right)_{\mathrm{s}^{\prime}}$. As monolayer(s) of $\mathrm{Ag}$ begin to form at the Pt electrode (in parallel to the dissolution of the dendrites at the Ag electrode), the emf increases again simultaneously and relatively fast because of $\left(a_{\mathrm{Ag}}\right)_{\mathrm{s}^{\prime}} \approx\left(a_{\mathrm{Ag}}\right)_{\mathrm{s}^{\prime \prime}}$. But owing to slower diffusion of the $\mathrm{Ag}^{+}$ions $a\left(\mathrm{Ag}^{+}\right)_{\mathrm{s}^{\prime}}>a\left(\mathrm{Ag}^{+}\right)_{\mathrm{s}^{\prime \prime}}$ the positive diffusion potential governs $V_{\text {cell }}$. Thus, the control over $V_{\text {cell }}$ changes from a situation mainly determined by equation (11) to a situation dominated by equation (5).

Emfs for different types of VCM and ECM memory cells. In the course of our emf studies, we investigated a series of different ECM cells as well as selected VCM cells. We were able to prove that in all types of ReRAM cells tested significant (electro)chemical potential gradients are generated by the operation of the cells. Inevitably, these gradients give rise to an emf, and, hence, the cells show the characteristics of nanosize batteries with cell voltages in the range displayed in Fig. 4a (for details see Supplementary Table S1). After forming, the cell voltages of VCM cells are much lower than for ECM cells owing to the significantly higher electronic conductance in the OFF state originating from the stub of the highly conductive filament.

\section{Discussion}

The non-equilibrium states reflected by the emf voltage may affect both the retention and the device operation. Although OFF 
a

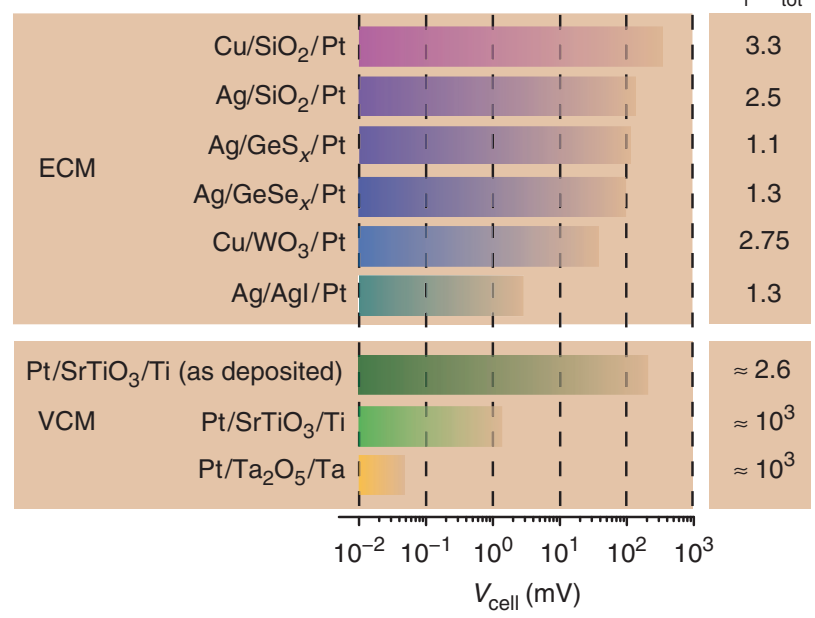

b

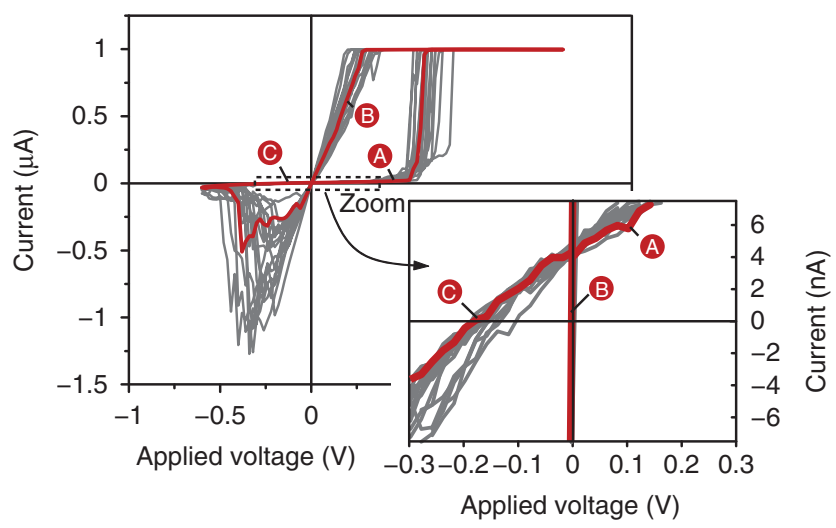

Figure 4 | Emf for different types of ReRAM cells. All tested cells showed an emf varying from some hundreds of microvolts to some hundreds of millivolts (a). The specific steady-state emf depends on the type of cell and the device operation (for example, sweep rate or voltage amplitude before the (open cell) emf measurement). For the sake of completeness, the internal resistance $R_{\mathrm{i}}$ normalized by the total resistance $R_{\text {tot }}$ is given (for details: see Supplementary Table S1). The influence of the emf on the current-voltage sweeps, resulting in non-zero-crossing characteristics (b), is given for the $\mathrm{Cu} / \mathrm{SiO}_{2} / \mathrm{Pt}$ system (red curve) as an example (electrode area $A_{\text {cell }}=2 \times 10^{-4} \mathrm{~cm}^{2}$, sweep rate $v=400 \mathrm{mVs}-1$ ). The $I-V$ characteristics in grey are provided for statistical verification. For the sake of clarity, the currents of the OFF state (A) and (C) and the ON state (B) are labelled, respectively. The formed valence change memory (VCM)-type cells ( $\mathrm{Ti} / \mathrm{SrTiO}_{3} / \mathrm{Pt}$ and $\mathrm{Ta} / \mathrm{Ta}_{2} \mathrm{O}_{5} / \mathrm{Pt}$ ) show lower emf for the OFF state owing to the higher electronic partial conductivity, that is, higher currents, but lower ion transference number. Emfs for VCM cells are depicted in Supplementary Figs S7,S8, respectively. The $1-\mathrm{V}$ characteristics for $\mathrm{Ag} / \mathrm{SiO}_{2} / \mathrm{Pt}$ and $\mathrm{Ti} / \mathrm{SrTiO}_{3} / \mathrm{Pt}$ cells are shown in Supplementary Figs $\mathrm{S} 9$, S10.

and intermediate states always experience emf voltages, for ON states with metallic contact the cell voltage is generally close to zero owing to the electronic conductance of the filament. Apart from that, chemical potential gradients, size effects and electrolyte non-stoichiometry can result in a chemical dissolution of the filament ${ }^{30}$. Thus, the retention of the $\mathrm{ON}$ state is strongly dependent on the filament characteristics and only indirectly dependent on the emf voltage.

Being an intensive state property, $V_{\mathrm{emf}}$ is independent on the cell size. However, the ionic and electronic resistances, $R_{\mathrm{i}}$ and $R_{\mathrm{e}}$, and hence $V_{\text {cell }}$ may scale in a non-trivial manner depending on the ReRAM type, for example, the properties of the filament stub and the gap between the filament tip and the electrode in the case of VCM cells. This is further illustrated in Supplementary Figs S5,S6.

Our results strongly suggest that in all bipolar ReRAM devices a nanobattery with dedicated emf voltages is present. This fact has far-reaching consequences on the application of the theory of memristive elements and on the device modelling in general. The emf is a state property corresponding to a non-zero-crossing $I-V$ characteristic (compare Fig. 4b), and we correspondingly expanded the memristive equations to obtain an active device that can be considered an extended memristive element. In fact, any electrochemical system is active by nature, thus two-terminal nanoionic-resistive switches cannot be pure passive memristors. The same is true for neurobiological systems, which are also active, offering passive memristive elements only as internal elements ${ }^{31}$. However, in contrast to the emf of ReRAM cells, the biological resting membrane potential is modulated only by $V_{\mathrm{d}}$ (eq. (5)) and not by $V_{\mathrm{N}}$ (eq. (3)). By abandoning the zero-crossing property, a large number of dynamical devices can be added to the framework of memristive, memcapacitive and meminductive devices. For example, ferroelectric capacitors as well as ferromagnetic inductors can thus be regarded as extended memcapacitive and meminductive elements, respectively (Fig. 5a). By means of this expanded framework, a ReRAM cell is still a memristive device, as shown in Fig. 5a.
The starting point for a practical memristive model of a ReRAM cell is the memristive model from Strukov et al. ${ }^{6}$, to which we add the nanobattery in parallel. The resulting model, which we term an extended memristive model, consists of a voltage source $V_{\text {emf }}$ and a nonlinear internal resistance $R_{\mathrm{i}}$-which together form the nanobattery-and a parallel resistor $R_{\mathrm{el}}$ whose state variable $x$ is controlled by the nanobattery (Fig. $5 \mathrm{~b}$ and Supplementary Fig. S11). The state-dependent resistance of the electronic current path $\left(R_{\mathrm{el}}\right)$ is a nonlinear function of the applied voltage, for example a tunnelling equation ${ }^{32}$. The electronic leakage current is accounted by a further parallel resistance $R_{\text {leak}}$, which is state-independent and already present in the pristine device. Both contributions, $R_{\mathrm{el}}$ and $R_{\text {leak }}$, in parallel represent $R_{\mathrm{e}}$ in Fig. 2a. The resistance of the ionic current path $\left(R_{\mathrm{i}}\right)$ is defined by another nonlinear equation, determined by the Butler-Volmer equation and/or the high-field drift equation. The statedependent Ohm's law for the extended memristive device reads:

$$
I=I_{\text {ion }}\left(V_{\mathrm{emf}}, V\right)+I_{\mathrm{el}}(x, V)=G(x, V) \cdot\left(V-t_{\text {ion }} V_{\mathrm{emf}}\right)
$$

and the state equation (compare Fig. 5b) reads:

$$
\dot{x}=K_{1} \cdot I_{\text {ion }} \text { with } 0 \leq x \leq d
$$

where $d$ is the thickness of the active layer and $K_{1}$ is a constant while $I_{\text {ion }}$ offers a highly nonlinear voltage dependence, responsible for the pronounced nonlinearity of the switching kinetics.

To fit the observed changes in the emf measurements (Fig. 2), a second-state variable, the ion concentration $c_{\text {ion }}$, is required. In this case, the emf equation (10) with the experimentally determined $V_{0}=0.17 \mathrm{~V}$ for $\mathrm{Ag} / \mathrm{SiO}_{2} / \mathrm{Pt}$ (Fig. 2c) reads:

$$
V_{\mathrm{emf}}=V_{0}+\frac{k T}{2 e} \ln \left(\frac{c_{\text {ion }}}{c_{0}}\right)
$$

Modelling details are described in the Supplementary Note 4 and corresponding simulation results are depicted in Fig. 5c. The inset clearly shows the non-zero-crossing $I-V$ behaviour. 
a

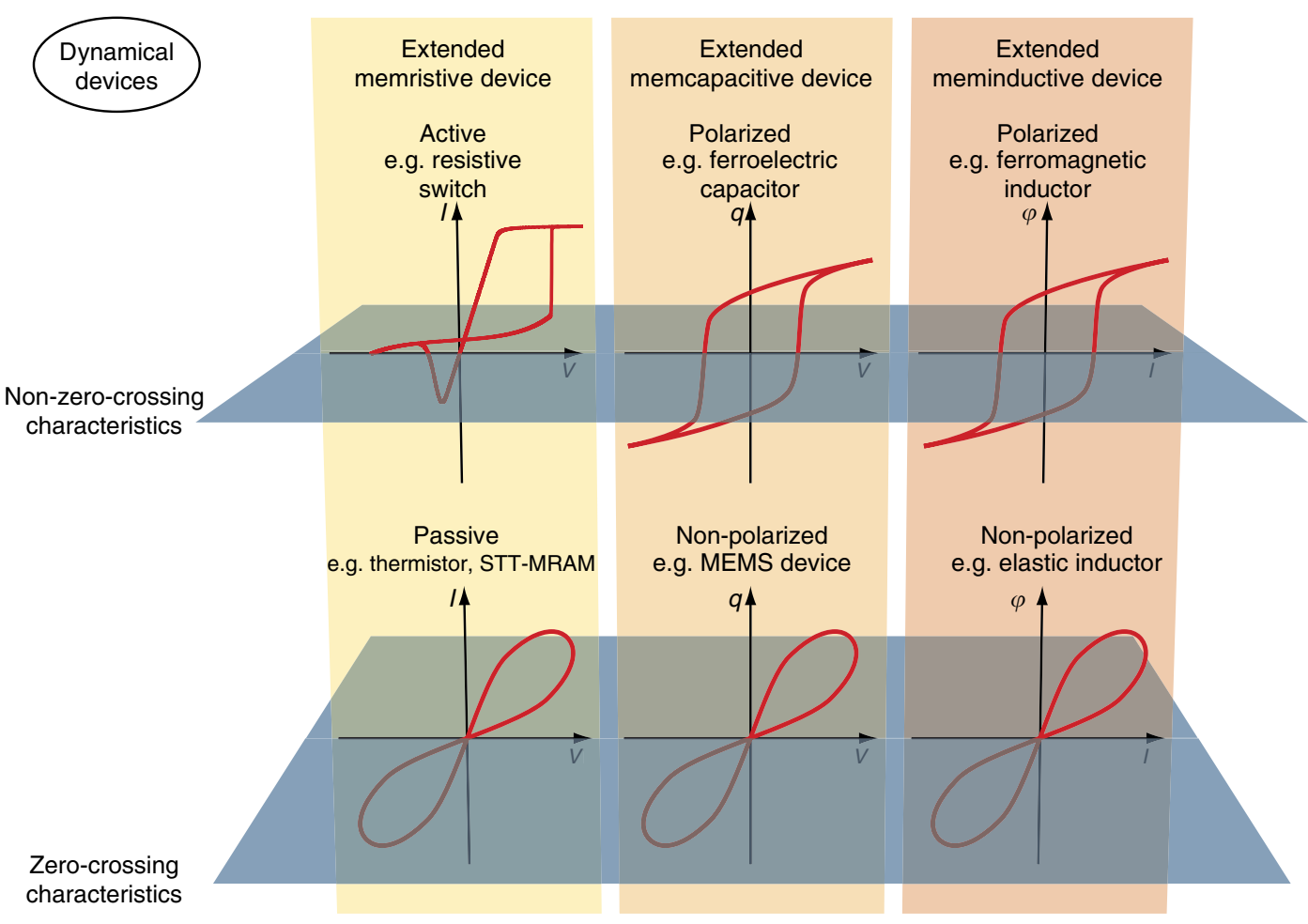

b

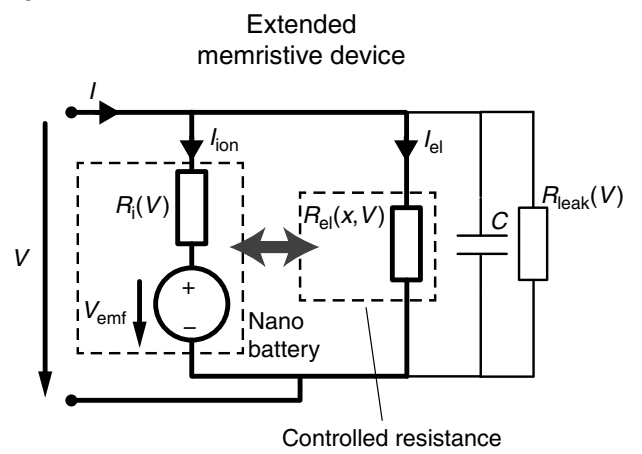

C

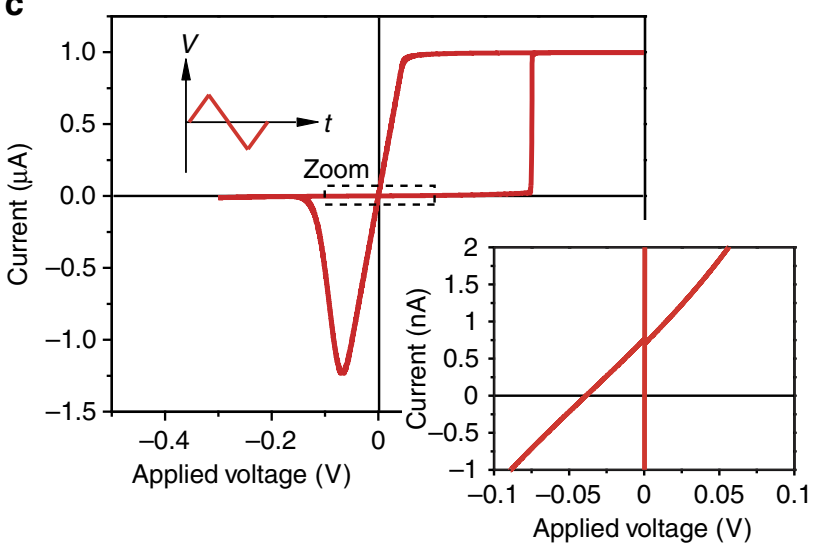

Figure 5 | Classification of mem devices. (a) Memristive, memcapacitive and meminductive devices are assumed to offer pinched characteristics at the origin in general ${ }^{39}$. In the case of memcapacitive and meminductive devices, a spontaneous polarization of a ferroelectric and ferromagnetic material, respectively, leads for example, to non-zero-crossing characteristics ${ }^{11}$. Similarly, a non-zero-crossing $I-V$ characteristic of a memristive device indicates the presence of an inherent nanobattery, that is, this device is active. Therefore, we introduce the generic terms extended memristive device, extended memcapacitive device and extended meminductive device to account for both zero-crossing and non-zero-crossing $I-V$ characteristics. Note that the origin of non-zero-crossing behaviour in memcapacitive and meminductive devices is of completely different nature than in memristive devices, thus require specific modifications of the concept. Interestingly, spin-transfer torque (STT) MRAM cells offer zero-crossing in contrast to ReRAM cells, thus can be considered as conventional memristive device. (b) Equivalent circuit of the extended memristive element. The ionic current is defined by the nanobattery, which controls the state-dependent resistor representing the electronic current path. The capacitance of the device is neglected as its influence is not significant. The partial electronic conductivity in the electrolyte induces a state-independent resistance $R_{\text {leak }}$ due to a leakage current in parallel. (c) Simulated $I-V$ characteristic of the extended memristor. The zoom shows the non-zero-crossing behaviour. Further considerations on the simulation are described in Supplementary Note 4.

Thus, ReRAM cells are non-zero-crossing devices, and therefore, the original memristor theory must be significantly extended in order to accommodate redox-based resistive switching systems.

Besides modelling accuracy, the emf also has direct impact on future memory device development and corresponding circuitry. First, the stability of intermediate resistive states (for example, required in multilevel memory and neuromorphic applications) must be carefully considered in terms of the emf. Moreover, the effect of the emf within ultra-dense passive crossbar arrays may become relevant owing to possible device-to-device interactions. Second, the READ voltage of future generation memory elements will be in the range of 100 to $200 \mathrm{mV}$ (ref. 33). This voltage is in the same order of magnitude as the emf voltage of some of the ReRAM types studied here. Third, the READ current for a 
ReRAM cell in a memory matrix will be in order of $100 \mathrm{nA}$ and the length of the READ pulse $<100 \mathrm{~ns}$. Hence, during the READ operation, a total charge of $Q=100 \mathrm{nA} \times 100 \mathrm{~ns}=10 \mathrm{fC}$ will be transferred to or from a memory cell. As shown in our paper, the total nanobattery capacity for scaled ReRAM cells may easily be in the same order of magnitude or higher. These points demonstrate impressively the relevance of nanobattery effect for the performance and reliability of future ReRAM devices, and the necessity of including it in the device modelling. Apart from that, the emf allows for novel read-out approaches in ReRAM cells, for example, we suggested using the emf for non-destructive read-out of complementary resistive switches ${ }^{34}$. The theoretical implication has been raised in refs 35,36.

In conclusion, we found clear indications that non-equilibrium states occur in nanoionic ReRAM cells, which are generated by chemical processes such as the dissolution of the active electrode material into the electrolyte, by electrochemical processes and by charge redistribution during the operation of the cells. The resulting electromotive force suggests the presence of a nanobattery inside the memristive device as an inherent property of the system. The nature of ReRAM systems implies a mandatory extension of the memristor theory, in order to include the non-zero-crossing characteristics.

\section{Methods}

Sample preparation. All samples were prepared using platinised silicon wafers as substrates. The solid electrolytes/insulators were deposited on the substrate using e-beam or thermal evaporation or radio frequency (RF) sputtering.

$\mathrm{SiO}_{2}$. Thirty nanometre to $50-\mathrm{nm} \mathrm{SiO}_{2}$ films were deposited by electron beam evaporation at $10^{-4} \mathrm{~Pa}$ at a deposition rate of $0.01 \mathrm{~nm} \mathrm{~s}^{-1}$. Later, pattern transfer of top microelectrodes with a diameter of 100 to $250 \mu \mathrm{m}$ was done using conventional UV lithography. $\mathrm{Ag}(\mathrm{Cu})$ with a thickness of $30 \mathrm{~nm}$ was deposited by e-beam evaporation (evaporation speed $0.025 \mathrm{~nm} \mathrm{~s}^{-1}$ ) followed by a 100 -nm-thick DC-sputtered Pt layer to prevent chemical oxidation of the silver (copper) electrode in air.

$\mathrm{WO}_{3-x}$. Thirty-nanometre $\mathrm{WO}_{3-x}$ films were sputtered in Ar plasma at a pressure of $10 \mathrm{~Pa}$ with an $\mathrm{RF}$ power of $121 \mathrm{~W}$, using a $\mathrm{WO}_{3}$ target. Subsequently, $70-\mathrm{nm}$-thick $\mathrm{Cu}$ electrodes $50 \mu \mathrm{m}$ in diameter (UV lithography) were thermally evaporated.

$\mathrm{GeS}_{2.2}$. Fifty-nanometre $\mathrm{GeS}_{2.2}$ films were deposited by RF sputtering at a gas flow of 50 s.c.c.m., $20 \mathrm{~Pa} \mathrm{Ar}$ pressure and RF power of $20 \mathrm{~W}$. The S/Ge ratio was determined by EDX (Oxford Instruments ISI 300, at $20 \mathrm{kV}$ acceleration voltage). Ag electrodes, microstructured by UV lithography, with diameters between 10 and $100 \mu \mathrm{m}$ and a thickness of $100 \mathrm{~nm}$ were RF sputtered at a gas flow of 50 s.c.c.m., RF power of $50 \mathrm{~W}$ and $20 \mathrm{~Pa}$ pressure. The relatively high pressure in the deposition chamber allows the deposition of homogeneous thin films ${ }^{37}$.

$\mathrm{GeSe}_{2.3}$. Fifty to $70-\mathrm{nm} \mathrm{GeSe}_{2.3}$ films were sputtered at a gas flow of 40 s.c.c.m., process pressure of $7 \times 10^{-2} \mathrm{~Pa}$ and RF power of $13 \mathrm{~W}$. The deposition rate was $0.075 \mathrm{~nm} \mathrm{~s}^{-1}$. The preparation of the electrodes is identical to that described for $\mathrm{GeS}_{2.2}$.

AgI. Thirty to 50-nm AgI films were prepared by thermal evaporation. To avoid any impact of UV light and chemicals, the pattern transfer for the top electrode was done directly after subtractive pattern transfer of the Pt bottom electrode using reactive ion etching. A detailed description of the fabrication of $\mathrm{Ag} / \mathrm{AgI} / \mathrm{Pt}$ cells can be found in Tappertzhofen et al. ${ }^{18}$

$\mathrm{SrTiO}_{3}$. Seventy nanaometre of $\mathrm{Ti}$ was sputtered in argon plasma at an $\mathrm{RF}$ power of $20 \mathrm{~W}$, a pressure of $0.8 \mathrm{~Pa}$ and a substrate temperature of $200^{\circ} \mathrm{C}$, followed by the application of $8 \mathrm{~nm}$ of $\mathrm{SrTiO}_{3}$, using the same process parameters and a sintered ceramic target. The Pt top electrodes with a thickness of $30 \mathrm{~nm}$ were sputtered at a pressure of $0.8 \mathrm{~Pa}$ and RF power of $12 \mathrm{~W}$ for lower kinetic impact. All layers were deposited without breaking the vacuum to prevent contamination.

$\mathrm{Ta}_{2} \mathrm{O}_{5}$. Fifty nanaometre of $\mathrm{Ta}$ was sputtered in argon plasma at an RF power of $14 \mathrm{~W}$, a pressure of $0.8 \mathrm{~Pa}$ and a substrate temperature of $200{ }^{\circ} \mathrm{C}$. Subsequently, the Ta layer was oxidized using an argon-oxygen atmosphere with a ratio of 3:1 and an oxygen partial pressure of $100 \mathrm{~Pa}$, forming $\mathrm{a} \mathrm{Ta}_{2} \mathrm{O}_{5}$ surface layer with a thickness of $15-20 \mathrm{~nm}$. The Pt top electrodes with a thickness of $30 \mathrm{~nm}$ were sputtered at a pressure of $0.8 \mathrm{~Pa}$ and RF power of $12 \mathrm{~W}$ for lower kinetic impact. All layers were deposited without breaking the vacuum to prevent contamination.

Electrical characterization. The emf measurements were performed in a fourneedle electrode microprobe station equipped with micromanipulators to contact the sample electrodes and an optical microscope to visually monitor the surface. For potentiodynamic current-voltage measurements, we applied triangular voltage sweeps between -1.5 and $1.5 \mathrm{~V}$ at various sweep rates $\left(30 \mathrm{mV} \mathrm{s}^{-1}\right.$ to $\left.2 \mathrm{~V} \mathrm{~s}^{-1}\right)$ using a Keithley 6430 sub-femtoampere SourceMeter. Details of the measuring method can be found in refs 25,38 . Electromotive forces were measured using the 6430 SourceMeter with high input impedance $\left(>10^{14} \Omega\right)$, a Keithley 617 electrometer $(>200 \mathrm{~T} \Omega)$ and a Keithley $2636 \mathrm{~A}$ SourceMeter $\left(>10^{14} \Omega\right)$ for comparison. Throughout the paper, the right electrode in cells denoted $\mathrm{M}^{\prime} / \mathrm{I} / \mathrm{M}^{\prime \prime}$ was used as reference electrode for all measurements. We used triaxial cables and electrostatic shielding to avoid RFI effects. The offset voltage measured across a $10 \mathrm{M} \Omega$ resistor has been proven to be within the device specification (below $1 \mu \mathrm{V}$ accuracy). Details on the measurement resolution and accuracy are shown in Supplementary Fig. S12 and Supplementary Table S2, respectively.

\section{References}

1. Aono, M. \& Hasegawa, T. The atomic switch. Proc. IEEE 98, 2228-2236 (2010).

2. Valov, I., Waser, R., Jameson, J. R. \& Kozicki, M. N. Electrochemical metallization memories-fundamentals, applications, prospects. Nanotechnology 22, 254003 (2011).

3. Waser, R., Dittmann, R., Staikov, G. \& Szot, K. Redox-based resistive switching memories-nanoionic mechanisms, prospects, and challenges. Adv. Mater. 21, 2632-2663 (2009).

4. Borghetti, J., Snider, G. S., Kuekes, P. J., Yang, J. J., Stewart, D. R. \& Williams, R. S. 'Memristive' switches enable 'stateful' logic operations via material implication. Nature 464, 873-876 (2010)

5. Fölling, S., Türel, Ö. \& Likharev, K. Single-electron latching switches as nanoscale synapses. Proc. IJCNN '01 216-221 (2001).

6. Strukov, D. B., Snider, G. S., Stewart, D. R. \& Williams, R. S. The missing memristor found. Nature 453, 80-83 (2008).

7. Waser, R. \& Aono, M. Nanoionics-based resistive switching memories. Nat Mater. 6, 833-840 (2007).

8. Chua, L. O. Memristor-the missing circuit element. IEEE Trans. Circuit Theory CT-18, 507-519 (1971).

9. Chua, L. O. \& Kang, S. M. Memristive devices and systems. Proc. IEEE 64, 209-223 (1976).

10. Ohno, T., Hasegawa, T., Tsuruoka, T., Terabe, K., Gimzewski, J. K. \& Aono, M. Short-term plasticity and long-term potentiation mimicked in single inorganic synapses. Nat. Mater. 10, 591-595 (2011).

11. Pershin, Y. V. \& Di Ventra, M. Memory effects in complex materials and nanoscale systems. Adv. Phys. 60, 145-227 (2011).

12. Yang, J. J., Strukov, D. B. \& Stewart, D. R. Memristive devices for computing. Nat. Nanotechnol. 8, 13-24 (2013).

13. Mazumder, P., Kang, S. \& Waser, R. Memristors: devices, models, and applications-scanning the issue. Proc. IEEE 100, 1911-1919 (2012).

14. Valov, I. et al. Atomically controlled electrochemical nucleation at superionic solid electrolyte surfaces. Nat. Mater. 11, 530-535 (2012).

15. Linn, E., Rosezin, R., Kügeler, C. \& Waser, R. Complementary resistive switches for passive nanocrossbar memories. Nat. Mater. 9, 403-406 (2010).

16. Yang, B., Liang, X. F., Guo, H. X., Yin, K. B., Yin, J. \& Liu, Z. G. Characterization of $\mathrm{RbAg}_{4} \mathrm{I}_{5}$ films prepared by pulsed laser deposition. J. Phys. D-Appl. Phys. 41, 115304 (2008).

17. Valov, I. \& Kozicki, M. N. Cation-based resistance change memory. J. Phys. D Appl. Phys. 46, 074005 (2013).

18. Tappertzhofen, S., Valov, I. \& Waser, R. Quantum conductance and switching kinetics of AgI based microcrossbar cells. Nanotechnology 23, 145703 (2012).

19. Yao, J. et al. Resistive switching in nanogap systems on $\mathrm{SiO}_{2}$ substrates. Small $\mathbf{5}$, 2910-2915 (2009).

20. Sakamoto, T., Lister, K., Banno, N., Hasegawa, T., Terabe, K. \& Aono, M. Electronic transport in $\mathrm{Ta}_{2} \mathrm{O}_{5}$ resistive switch. Appl. Phys. Lett. 91, 092110 (2007).

21. Jameson, J. R. et al. One-dimensional model of the programming kinetics of conductive-bridge memory cells. Appl. Phys. Lett. 99, 063506 (2011).

22. Russo, U., Kamalanathan, D., Ielmini, D., Lacaita, A. L. \& Kozicki, M. N. Study of multilevel programming in programmable metallization cell (PMC) memory. IEEE Trans. Electron Devices 56, 1040-1047 (2009).

23. Chua, L. O. Resistance switching memories are memristors. Appl. Phys. A-Mater. Sci. Process 102, 765-783 (2011).

24. Vetter, KJ. Electrochemical Kinetics (Springer Verlag, 1961).

25. Tappertzhofen, S., Mündelein, H., Valov, I. \& Waser, R. Nanoionic transport and electrochemical reactions in resistively switching silicon dioxide. Nanoscale 4, 3040-3043 (2012). 
26. Tsuruoka, T., Terabe, K., Hasegawa, T., Valov, I., Waser, R. \& Aono, M. Effects of moisture on the switching characteristics of oxide-based, gapless-type atomic switches. Adv. Funct. Mater. 22, 70-77 (2012).

27. Miao, F., Yang, J. J., Borghetti, J., Medeiros-Ribeiro, G. \& Williams, R. S Observation of two resistance switching modes in $\mathrm{TiO}_{2}$ memristive devices electroformed at low current. Nanotechnology 22, 254007 (2011).

28. Choi, S., Balatti, S., Nardi, F. \& Ielmini, D. Size-dependent drift of resistance due to surface defect relaxation in conductive-bridge memory. IEEE Electron Device Lett. 33, 1189-1191 (2012).

29. Mitkova, M., Kozicki, M. N., Kim, H. C. \& Alford, T. L. Thermal and photodiffusion of Ag in S-rich Ge-S amorphous films. Thin Solid Films 449, 248-253 (2004).

30. Cho, D.Y., Valov, I., van den Hurk, J., Tappertzhofen, S. \& Waser, R. Direct observation of charge transfer in solid electrolyte for electrochemical metallization memory. Adv. Mater. 24, 4552-4556 (2012).

31. Chua, L., Sbitnev, V. \& Kim, H. Hodgkin-Huxley axon is made of memristors. Int. J. Bifurcat. Chaos 22, 1230011 (2012).

32. Menzel, S., Böttger, U. \& Waser, R. Simulation of multilevel switching in electrochemical metallization memory cells. J. Appl. Phys. 111, (2012).

33. ITRS. The International Technology Roadmap for Semiconductors-ITRS 2011 Edition 2011.

34. Rosezin, R. et al. Verfahren zum nichtdestruktiven Auslesen resistiver Speicherelemente und Speicherelement. German Patent DE102011012738 (2012).

35. Meuffels, P. \& Soni, R. Fundamental Issues and Problems in the Realization of Memristors. Preprint at http://arxiv.org/abs/1207.7319 (2012).

36. Saraf, S., Markovich, M., Vincent, T., Rechter, R. \& Rothschild, A. Memory diodes with nonzero crossing. Appl. Phys. Lett. 102, 22902 (2013).

37. van den Hurk, J., Valov, I. \& Waser, R. Preparation and characterization of $\mathrm{GeS}_{\mathrm{x}}$ thin-films for resistive switching memories. Thin Solid Films 527, 299-302 (2012).

38. Bard, A. \& Faulkner, L. Electrochemical Methods: Fundamentals and Applications (John Wiley and Sons, New York, 2001).

39. Di Ventra, M., Pershin, Y. V. \& Chua, L. O. Circuit elements with memory: memristors, memcapacitors, and meminductors. Proc. IEEE 97, 1717-1724 (2009).

\section{Acknowledgements}

We gratefully acknowledge financial support in parts by the Deutsche Forschungsgemeinschaft through the SFB 917 'Nanoswitches' and the project WA 908/22, as well as by Intel Corp and by Samsung Advanced Institute of Technology (SAIT-GRO Program). We would like to thank S. Menzel for fruitful discussions on the kinetics of the resistive switching, and R. Meyer for efficient proof reading and excellent comments. The supply of $\mathrm{Si}$ wafers with nanostructured $\mathrm{TiN}$ bottom electrodes by IMEC, Leuven, for our study shown in Supplementary Fig. S6 is gratefully acknowledged.

\section{Author contributions}

I.V. conceived the idea, designed the experiments, interpreted the data and wrote the manuscript; E.L. performed the memristive simulations, co-wrote the manuscript and contributed to data interpretation; S.T. prepared the ECM cells, performed the measurements and contributed to data interpretation; S.S. performed measurements on VCM cells; J.v.d.H. prepared $\mathrm{GeS}_{x}$ cells; F.L. prepared the $\mathrm{WO}_{x}$ cells and performed measurements; R.W. initiated and supervised the research, and contributed to the concept of the study. All authors discussed the results and implications at all stages, and contributed to the improvement of the manuscript text.

\section{Additional information}

Supplementary Information accompanies this paper at http://www.nature.com/ naturecommunications

Competing financial interests: The authors declare no competing financial interests.

Reprints and permission information is available online at http://npg.nature.com/ reprintsandpermissions/

How to cite this article: Valov, I. et al. Nanobatteries in redox-based resistive switches require extension of memristor theory. Nat. Commun. 4:1771 doi: 10.1038/ncomms2784 (2013).

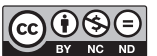

This work is licensed under a Creative Commons AttributionNonCommercial-NoDerivs 3.0 Unported License. To view a copy of this license, visit http://creativecommons.org/licenses/by-nc-nd/3.0/ 